\title{
AUTOSOMAL DOMINANT POLYCYSTIC KIDNEY DISEASE WITH HEPATIC CYSTS COMPLICATIONS IN A HEMODIALYSIS PATIENT: A CASE REPORT
}

\author{
Fasie Dragos', Cimpineanu Bogdan' , Catalina Oana1, Gheorghita Raluca1, Ion Ileana1
}

${ }^{1}$ Faculty of Medicine, University "Ovidius" of Constanta

Ileana Ion

Faculty of Medicine, Univeristy ,Ovidius” of Constanta,

Universitatii Alee No. 1, Campus B, Constanta, Romania email:ileanaion58@yahoo.com

\begin{abstract}
Background: Autosomal dominant polycystic kidney disease (ADPKD) is the most common inherited kidney disease in humans. It is a multisystemic disorder characterized by progressive cystic dilatation of both kidneys, with variable extrarenal manifestations in the gastrointestinal tract (hepatic cysts), cardiovascular system, reproductive organs and brain. An important issue of patients with hepatorenal cystic disease is the fact that complications can arise due to the growing of the cysts: local kidney complications (intracystic infection, intracystic hemorrhage) and local liver complications (portal hypertension as a result of portal vein compression by cysts, bile duct compression, ruptures and bleedings of the cysts, obstruction of the liver veins).

Objective: The main purpose of our case presentation is to emphasize the fact that ADPKD can be an aggressive disease with multiple complications, which requires an early diagnosis in order to properly avoid possible complications.

Material and Method: We present the case of a 61 years old male, known with polycystic hepatorenal disease, chronic kidney disease (CKD) in chronic hemodialysis since 2010, renal hypertension, hypertensive cardiomyopathy, renal anemia and stage 1 chronic lymphatic leukemia. The patient was diagnosed with hepatorenal polycystic disease 20 years ago and 9 years ago he started undergoing renal replacement therapy by hemodialysis. Three months ago, the patient presented a suddenly installed ascitic syndrome for which an MRI was performed. The imagistic investigation revealed multiple kidney and liver cysts, with secondary compression of the portal vein and a tumoral mass that may suggest an adenocarcinoma.

Results: The investigations performed confirm the diagnosis of portal hypertension secondary to compression due to cystic formations.

Conclusions: Hepatorenal polycystic disease has numerous clinical variations, so it needs to be followed in a multidisciplinary way with rapid therapeutic measures to prevent complications. Further investigations are needed when dealing with suspicious cystic formations with an uncertain substrate.

Keywords: ADPKD, hepatorenal disease, multisystemic disorder, dialysis
\end{abstract}

\section{INTRODUGTION}

Autosomal dominant polycystic kidney disease (ADPKD) is the most common inherited kidney disease in humans. It is a multisystemic disorder characterized by relentless cystic dilatation and enlargement of both kidneys, with variable extrarenal manifestations in the gastrointestinal tract (hepatic cysts), cardiovascular system, reproductive organs and brain. The genes responsible for ADPKD are polycystin 1 (PKD1) and polycystin 2 (PKD2) genes. PKD 1 is localized at the short arm of chromosome 16 and it is involved in $85 \%$ of cases of polycystic renal disease, while PKD2 is localized at the long arm of chromosome 4 , being involved in most of the remaining cases.

Worldwide, ADPKD affects approximately 4 to 7 million individuals. In $50 \%$ of cases the disease progresses to chronic terminal renal failure (CKD) around the age of 55 years, these patients requiring dialysis or kidney transplantation. In fact, $A D P K D$ is responsible for about $10 \%$ of cases of CKD. $(1,2)$ In dialyzed ADPKD patients, the 
most frequent complications are cardiovascular, followed by those related to the vascular access (central venous catheter or arterio-venous fistula), and last, but not least, secondary to extrarenal manifestations of polycystic disease (3)

The most important extrarenal manifestation of ADPKD is PLD (polycystic liver disease), which does not affect liver function, but represents a heterogeneous set of structural changes of the biliary tree development and generate symptoms related to mass effects when significant liver enlargement occurs (4). Often these cysts are clinically insignificant, they representing incidental findings. The frequency of hepatic cysts increases with age in women, especially those under hormonal influence such as estrogen replacement therapy or pregnancies, and are rare in children (5). The hepatic cysts epithelial cells present estrogen receptors and growth factor similar with IGF-1 (insulin-like growth factor 1) that when stimulated promotes proliferation and cystic growth $(6,7)$.

The majority of the patients with ADPKD report no liver symptoms, some experience chronic manifestations related to progressive increase of the liver cysts (8). In most cases, PLD is related to ADPKD and can be linked either to PKD1 (most frequent) or to PKD2. The isolated form of autosomal dominant PLD has been firmly established as a distinct inherited disorder unlinked to PKD1 or PKD2 (9).

An important issue of patients with hepato-renal cystic disease is the complication that can arise due to the growing of the cysts: local kidney complications (intracystic infection, intracystic bleeding) and local liver complications (portal hypertension, bile duct compression, ruptures and bleedings of the cysts, obstruction of the liver veins).

We report the case of a 61 years old male, known with ADPKD diagnosed 20 years ago, stage $\mathrm{V}$ CKD, in chronic HD (hemodialysis) program for 9 years, hypertensive with hypertensive cardiopathy for which he is under chronic treatment with Furosemide $40 \mathrm{mg}$ / day on day without HD, Nebilet 5mg, $1 \mathrm{cp} /$ day, Aspenter $75 \mathrm{mg} /$ day on day without HD, stage 1 CLL, mixed secondary anemia for which he is under an erythropoiesis stimulating agent treatment (Aranesp $20 \mathrm{mcg} / \mathrm{month}$ ), Iron complex 100mg, 1cp/day, Folic Acid $5 \mathrm{mg}, 2 \mathrm{cp} /$ day, hyperuricemia for which he is under Allopurinol $300 \mathrm{mg}, 1 / 2 \mathrm{cp} /$ day treatment.

Two months ago, he presented repeated episodes of abdominal colic and bloating sensation which did not subside under antalgic therapy. In the last month the patient reports an increase in abdomen volume.

The physical examination reveals pale skin and mucosa, non-palpable peripheral lymph nodes, hypotrophy of the muscle tissue, cone shaped thorax, bilateral vesicular murmur present, without rales, hemodynamically stable, $\mathrm{ABP}=$ $140 / 70 \mathrm{mmHg}$, rate $=70 /$ minute, abdominal distension, with movable dullness in the flanks, no pain at abdominal palpation, inferior limit of the liver at $5 \mathrm{~cm}$ under the costal margin, small umbilical hernia, painless, retractable, third degree splenomegaly. Giordano maneuver negative bilaterally, no pain was present in the ureteral points.

The biological assays reveal:

- biochemical: alkaline reserve: $22 \mathrm{mEq} /$ 1CO2; serum creatinine: $9.14 \mathrm{mg} / \mathrm{dl}$; serum urea: $139 \mathrm{mg} / \mathrm{dl}-$ predialysis; serum uric acid: $5.7 \mathrm{mg} / \mathrm{dl}$; serum calcium: $8.3 \mathrm{mg} / \mathrm{dl}$; serum phosphor: $2.7 \mathrm{mg} / \mathrm{dl}$; serum potassium: $5.5 \mathrm{mg} /$ dl; serum glucose: $96 \mathrm{mg} / \mathrm{dl}$; AST: 23 U/1; ALT: $19 \mathrm{U} / 1$; carcinoembryonic antigen: $5 \mathrm{ng} / \mathrm{dl}$.

- hematology: WBC: $66270 / \mu \mathrm{L}$; RBC: $2.9 \mathrm{mil} / \mu \mathrm{L}$; platelets: 150000/ $\mu \mathrm{L}$; hemoglobin: 8,9 g/dl; hematocrit: 30.2\%; MCV: 104. 1 fL; MCH: 30.7 pg/ cell; neutrophils: $8.1 \%$; lymphocytes: $87.3 \%$; serum iron: $40 \mathrm{mcg} / \mathrm{dl}$; transferrin: $1.3 \mathrm{~g} / \mathrm{L}$

Stage diagnosis being a newly installed ascitic syndrome, it is decided to perform an exploratory paracentesis. The results shows:

- cytology: numerous inflammatory cells, rare red blood cells

- biochemistry: serum albumin: $2.11 \mathrm{~g} /$ $\mathrm{dl}$; ascites albumin: $3.24 \mathrm{~g} / \mathrm{dl}$ (SAAG $<1.1 \mathrm{~g} / \mathrm{dl})$

- bacteriology: no pathogens germs have been identified.

An abdominal ultrasonography has been performed (Figure 1), this indicates: numerous 
liver cystic formations (maximum $7.3 \mathrm{~cm}$ ); a 5.8 $\mathrm{cm}$ cystic formation in the hepatic hilum with compressive mass effect on the portal vein (PV), CBD (common bile duct): $6 \mathrm{~cm}$; splenic vein: $10 \mathrm{~mm}$; moderate amount of ascites fluid.

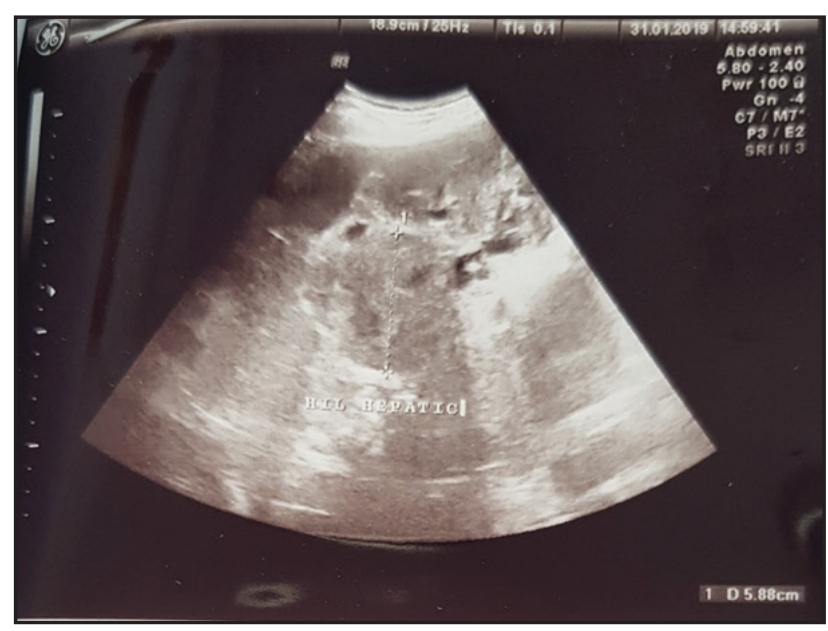

Figure 1. Patient Ultrasound

Following imagistic investigations and the results of the exploratory paracentesis, we can say that the etiology of the ascitic syndrome is uncertain.

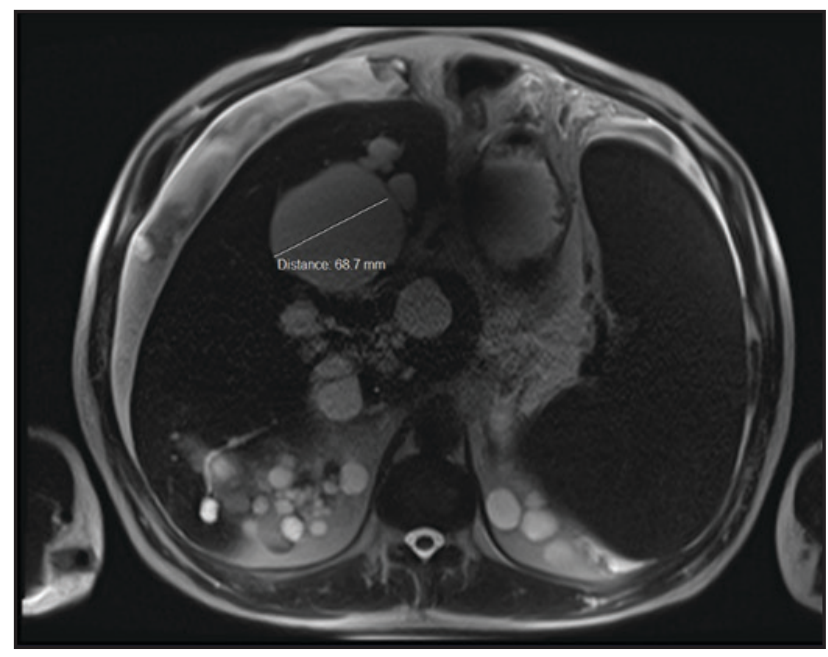

Figure 2. MRI aspect of the cysts in the hepatic hilum

Due to the uncertainultrasonographic aspect of the hepatic hilum formation (complicated hepatic cyst or a tumoral mass) it is decided to perform an MRI with contrast agent which revealed: a space replacing formation (Figure 2 and 3) of $6.8 / 6.4 \mathrm{~cm}$ in T1 and T2 hypersignal, gadophil in arterial phase, without "wash-out" phenomena, developed in the segment IV of the liver, left branch of PV thrombosis, without
IHBD (intrahepatic biliary ducts) and EHBD (extrahepatic biliary ducts) dilatations, normal aspect of the pancreas, spleen with $21.1 \mathrm{~cm}$ diameter, LK and RK with present secretion, celiac trunk adenopathy (maximum $2.1 \mathrm{~cm}$ ), significant amount of fluid in the peritoneal cavity at the level of the examined segment.

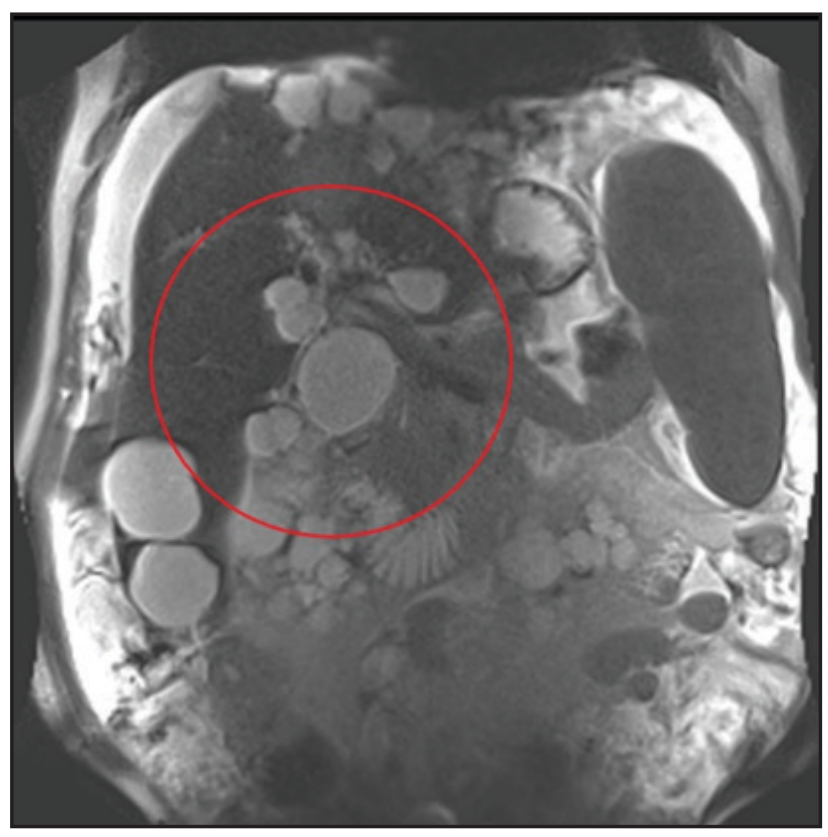

Figure 3. Complicated hepatic cyst

Although the initial ultrasonographic aspect argues more for a tumoral pathology, the final diagnosis is: complicated hepatic cyst localized in segment IV of the liver, with modified content (hematic or protein), which causes compression on the left branch of the portal vein, with portal hypertension installation and an impressive amount of ascitic fluid.

After the imagistic investigations, the evacuation of the ascitic fluid is decided, with an initial favorable evolution, but after about 2 weeks the patient returns with the same symptomatology (discomfort and abdominal pain) and the increase in abdominal volume. A new evacuation paracentesis is performed.

Because the evolution of the patient was not good, with the rapid recovery of de ascites fluid, it is decided to perform an echoendoscope guidance puncture of the cystic formations with mass effect on the portal vein. Endoscopic ultrasound fine needle aspiration (EUS-FNA) of the cyst was performed, the cystic formations being localized in the hepatic hilum. Reaching 
the gastric level, the echoendoscope was able to fully view the left hepatic lobe occupied entirely by numerous cystic formations of different dimensions, at the level of the hepatic hilum the portal vein is compressed by two cysts with thin wall and transonic content, with $78 / 69 \mathrm{~mm}$ diameter. Afterwards a puncture was made with a Boston fine needle at the level of a cyst that causes compression on the portal vein, with the release of $100 \mathrm{ml}$ of serocytrin fluid, without incidents and rapidly favorable later evolution of the patient.

\section{Discussions}

The majority of the hepatic cysts are asymptomatic. When the symptomatology occurs, can be due to mass effect, hemorrhage, rupture or infection of the cysts (10) and can include abdominal pain, vomiting, nausea, early satiety, hepatomegaly and obstructive jaundice $(10,11)$. The management of the hepatic cyst formations has varied over the years. The therapeutic options include the surgical approach (such as open or laparoscopic deroofing, complete resection of the cysts and hepatectomy), sclerotherapy and percutaneous aspiration $(10,11,12-15)$. Previous reports have shown that percutaneous aspiration is associated with a very high recurrence rate (nearly to $100 \%$ ) observed earlier than 2 weeks $(13,15)$. The percutaneous approach gave better results for treating right liver lobe cysts, while for the left liver lobe cysts EUS-FNA is the most efficient method (16).

Endoscopic echography (EUS) represents a technique that uses ultrasound during an endoscopic procedure to look at or through the wall of gastro-intestinal tract. This procedure allows the visualization of the organs and structures that are usually not visible during gastro-intestinal endoscopy such as the layers of the gastrointestinal tract wall, the liver, bile ducts, pancreas, and lymph nodes. The use of EUS is not limited to visualization, but also allows tissue biopsy sampling for diagnostic purpose. EUS has played a major role in revolutionizing the diagnosis of the focal liver lesions (17).

The role of EUS has evolved greatly in recent years. Initial was considered to be an excellent diagnostic tool, but currently EUS has several therapeutic implications. With the implication of the EUS in liver disorders, the tool has proved to be excellent for gastroenterologists and hepatologists in managing more liver related conditions. Focal liver lesions have always been a challenge for the hepatologists. Along with progress in EUS, the tool has demonstrated superiority in detecting focal liver lesions compared to conventional scanning techniques such as CT or ultrasonography (18).

Recent data is showing that EUS guided liver biopsy can be safer than percutaneous liver biopsy, especially when done by experienced endosonographer (18).

In regards of portal hypertension, EUS can detect early changes in portal hypertension and therefore provide an accurate and early assessment of global clinical status (18).

The particularity of the case is given by the presence of multiple hepatic cystic formations within the ADPKD, in a male patient (liver cysts are usually more frequent in females due to increased concentrations of estrogen hormones), the cystic formations being localized predominantly at the level of the hepatic hilum causing compression on the portal vein, thus installing the portal hypertension.

The issue of ascitic syndrome etiology should always be discussed, in this case having contradictory data- the imagistic results which indicates portal hypertension due to cystic compressing on the portal vein and the results obtained from the exploratory paracentesis that indicate an exudate which leads towards another pathology.

\section{Conclusions:}

Hepatorenal polycystic disease has numerous clinical variations, so it needs to be addressed in a multidisciplinary way with rapid therapeutic measures to prevent complications.

Further investigations are needed when dealing with suspicious liver formations with an uncertain substrate. In those situations with advanced cases of hepatic cyst growth with compression on the portal system, endoscopic ecography and fine needle aspiration are imposed as the primary diagnostic and treatment choice. 


\section{References:}

1. Fedeles SV, Gallagher AR, Somlo S. Polycystin-1: a master regulator of intersecting cystic pathways. Trends Mol Med. 2014;20(5):251-60.

2. Lemaigre FP. Molecular mechanisms of biliary development. Prog Mol Biol Transl Sci. 2010;97:103-26.

3. Checheriţă IA, Tuţă LA, David C, Peride I, Niculae A. An overview of permanent vascular access in hemodialyzed patients. Rom J Morphol Embryol, 2015; 56 (1), 2731.

4. Raynaud P, Carpentier R, Antoniou A, Lemaigre FP. Biliary differentiation and bile duct morphogenesis in development and disease. Int $\mathrm{J}$ Biochem Cell Biol. 2011;43:245-56.

5. Abu-Wasel B, Walsh C, Keough V, Molinari M. Pathophysiology, epidemiology, classification and treatment options for polycystic liver diseases. World J Gastroenterol. 2013;19(35):5775-86.

6. Onori P, Franchitto A, Mancinelli R, Carpino G, Alvaro D, Francis H, et al. Polycystic liver diseases. Dig Liver Dis. 2010;42(4):261-71.

7. Alvaro D, Onori P, Alpini G, Franchitto A, Jefferson DM, Torrice A, et al. Morphological and Functional Features of Hepatic Cyst Epithelium in Autosomal Dominant Polycystic Kidney Disease. Am J Pathol. 2008;172(2):321-332.

8. Dominique Chauveau, Fadi Fakhouri, JeanPierre Grunfeld. Liver Involvement in Autosomal-Dominant Polycystic Kidney Disease-Therapeutic Dilemma

9. Pirson Y, Lannoy N, Peters D, Geubel A, Gigot JF, Breuning M, Verellen-Dumoulin C: Isolated polycystic liver disease as a distinct genetic disease, unlinked to polycystic kidney disease 1 and polycystic kidney disease 2. Hepatology 23:249 -252, 1996

10. Asuquo M, Nwagbara V, Agbor C, Otobo F, Omotoso A. Giant simple hepatic cyst: a case report and review of relevant literature. Afr Health Sci. 2015;15:293-298.

11. Srinivasan I, Tang SJ, Vilmann AS, Menachery J, Vilmann P. Hepatic applications of endoscopic ultrasound: Current status and future directions. World J Gastroenterol. 2015;21:12544-12557.

12. Mazza OM, Fernandez DL, Pekolj J, Pfaffen G, Sanchez Clariá R, Molmenti EP, de Santibañes E. Management of nonparasitic hepatic cysts. J Am Coll Surg. 2009;209:733739.

13. Saini S, Mueller PR, Ferrucci JT Jr, Simeone JF, Wittenberg J, Butch RJ. Percutaneous aspiration of hepatic cysts does not provide definitive therapy. AJR Am J Roentgenol. 1983;141:559-560.

14. Wijnands TF, Görtjes AP, Gevers TJ, Jenniskens SF, Kool LJ, Potthoff A, Ronot M, Drenth JP. Efficacy and Safety of Aspiration Sclerotherapy of Simple Hepatic Cysts: A Systematic Review. AJR Am J Roentgenol. 2017;208:201-207.

15. Maruyama Y, Okuda K, Ogata T, Yasunaga M, Ishikawa H, Hirakawa Y, Fukuyo K, Horiuchi H, Nakashima O, Kinoshita H. Perioperative challenges and surgical treatment of large simple, and infectious liver cyst - a 12-year experience. PLoS One. 2013;8:e76537.

16. Lee S, Seo DW, Paik WH, Park DH, Lee SS, Lee SK, Kim MH. Ethanol lavage of huge hepatic cysts by using EUS guidance and a percutaneous approach. Gastrointest Endosc. 2014;80:1014-1021.

17. Hammoud GM, Almashhrawi A, Ibdah JA. Usefulness of endoscopic ultrasound-guided fine needle aspiration in the diagnosis of hepatic, gallbladder and biliary tract Lesions. World J Gastrointest Oncol. 2014;6:420 429.

18. Hamzeh A Ssraireh, Mohammad Bilal, Shailendre Singh. Role of endoscopic ultrasound in liver disease: Where do we stand in 2017 ? 